\title{
Repurposing of FDA-Approved Toremifene to Treat COVID-19 by blocking the spike glycoprotein and NSP14 of SARS-CoV-2
}

William R. Martin ${ }^{1}$ and Feixiong Cheng ${ }^{1-3}$

${ }^{1}$ Genomic Medicine Institute, Lerner Research Institute, Cleveland Clinic, Cleveland, $\mathrm{OH}$ 44195, USA

${ }^{2}$ Department of Molecular Medicine, Cleveland Clinic Lerner College of Medicine, Case

Western Reserve University, Cleveland, OH 44195, USA

${ }^{3}$ Case Comprehensive Cancer Center, Case Western Reserve University School of Medicine, Cleveland, $\mathrm{OH} 44106$, USA

*To whom correspondence should be addressed:

Feixiong Cheng, $\mathrm{PhD}$

Lerner Research Institute, Cleveland Clinic

Tel: +1-216-444-7654; Fax: +1-216-636-0009

Email: chengf@ccf.org 


\section{ABSTRACT}

The global pandemic of Coronavirus Disease 2019 (COVID-19), caused by severe acute respiratory syndrome coronavirus 2 (SARS-CoV-2), has led to the death of more than 350,000 worldwide and over 100,000 in the United States alone. However, there are currently no proven effective pharmacotherapies for COVID-19. Here, we combine homology modeling, molecular docking, molecular dynamics simulation, and binding affinity calculations to determine potential targets for toremifene, a selective estrogen receptor modulator which we have previously identified as a SARS-CoV-2 inhibitor. Our results indicate the possibility of inhibition of the spike glycoprotein by toremifene, responsible for aiding in fusion of the viral membrane with the cell membrane, via a perturbation to the fusion core. An interaction between the dimethylamine end of toremifene and residues Q954 and N955 in heptad repeat 1 (HR1) perturbs the structure, causing a shift from what is normally a long, helical region to short helices connected by unstructured regions. Additionally, we found a strong interaction between toremifene and the methyltransferase non-structural protein (NSP) 14 , which could be inhibitory to viral replication via its active site. These results suggest potential structural mechanisms for toremifene by blocking the spike protein and NSP14 of SARS-CoV-2, offering a drug candidate for COVID-19. 


\section{INTRODUCTION}

As of June 1, 2020, there are over 6.2 million documented cases of COVID-19 (nearly 375,000 resulting in death), with nearly one-third of all cases occurring in the United States (over 100,000 deaths, source: Worldometers.info). As of this writing, there are no FDA approved treatments or vaccines for COVID-19, both of which are sorely needed. A search on clinicaltrials.gov for COVID-19 as the disease and the additional term "drug" yields 806 results, providing evidence for the need for an intervention. Drug repurposing allows an acceleration of the drug discovery pipeline; drugs which have already been FDA approved to treat another disease are repositioned as therapeutics for diseases for which they have not yet been used ${ }^{1,2}$.

In our initial network-based drug repurposing study ${ }^{3}$, we identified toremifene, another selective estrogen receptor modulator (SERM), as a strong candidate for the potential treatment of COVID-19. A drug repurposing study for SARS-CoV-14 indicated a low $50 \%$ effective concentration $\left(\mathrm{EC}_{50}\right)$ for toremifene, and noted that estrogen signaling may not be involved in the inhibitory pathway, similar to that of inhibition of ebola ${ }^{5}$. Indeed, a crystal structure of the Ebola virus with bound toremifene indicates the interaction lies between the attachment (GP1) and fusion (GP2) protein subunits ${ }^{6}$. In a study using human organs-on-chips ${ }^{7}$, toremifene was found to significantly inhibit entry of a pseudotyped SARS-CoV-2 virus. However, the mechanism of action for toremifene in the inhibition of SARS-CoV-2 is not yet known. As noted above, experimental studies have indicated a potential for toremifene to inhibit both Ebola virus and coronaviruses (Table 1). 
The actual target for toremifene has not yet been elucidated in coronaviruses as it has in Ebola, which was determined to have an $\mathrm{IC}_{50}$ of roughly $1 \mu \mathrm{M}^{5}$. Dyall et. al ${ }^{4}$ found the $\mathrm{EC}_{50}$ of toremifene for MERS-CoV and SARS-CoV-1 to be $12.9 \mu \mathrm{M}$ and $11.97 \mu \mathrm{M}$, respectively; while these results are not different, the story is different for tamoxifen, which differs from toremifene by a substitution of hydrogen for the chlorine on toremifene. While the $\mathrm{EC}_{50}$ for tamoxifen is slightly lower in MERS-CoV $(10.1 \mu \mathrm{M})$, the $\mathrm{EC}_{50}$ for SARS-CoV1 is worse than for toremifene $(92.9 \mu \mathrm{M})$, which could potentially indicate the inhibition is related to a viral, and not human, protein. Importantly, a recent study indicated an $\mathrm{IC}_{50}$ of $3.58 \mu \mathrm{M}$ for toremifene with SARS-CoV- $2^{8}$.

There have been numerous studies recently targeting singular proteins in SARSCOV-2 using virtual screening techniques, such as 3-chymotrypsin-like protease ${ }^{9-11}$ and the papain-like protease ${ }^{12}$. Further studies have been done using large databases, such as $\mathrm{ZINC}^{13}$, to test large compound libraries across multiple viral proteins. In an effort to determine potential interactors with toremifene specifically ${ }^{14}$, we used inverse virtual screening to test 13 of the 29 viral proteins encoded by the SARS-CoV-2 genome. Proteins which did not have a crystal structure at the time of this study were modeled using homology modeling. We believe this to be a comprehensive study to combine virtual screening with molecular dynamics and molecular mechanics / Poisson Boltzmann Surface Area (MM/PBSA) calculations to determine potential protein-ligand interactions in SARS-CoV-2 proteome. Here, we have discovered two potential targets for toremifene from the entire SARS-CoV-2 proteome. 


\section{EXPERIMENTAL PROCEDURES}

\subsection{Homology Modeling}

Proteins for which crystal structures were not available were constructed using homology modeling. Each sequence was accessed from NCBI by its accession number. The protein sequence was submitted to a BLAST ${ }^{15}$ search within UCSF Chimera ${ }^{16}$, and the best matching structure was chosen for homology modeling. Each homology model was constructed using a single template using MODELLER $9.21^{17}$ within UCSF Chimera. The best model as determined by Z-score was used for docking. Proteins which do not have potential templates with high homology were not modeled.

\subsection{System Equilibration}

Prior to docking, all homology models were subjected to a short minimization and equilibration period using molecular dynamics simulations. In short, each system was constructed using standard tools in GROMACS $2018.2^{18}$. Systems were submerged in a water box with edges no less than $10 \AA$ from any part of the protein, and neutralized using sodium and chloride ions to an ionic strength of $0.15 \mathrm{M}$. Parameterization for the protein, ions, and water were done using the CHARMM36 force field ${ }^{19}$. Each protein was minimized using a steepest descent algorithm for 5000 steps, followed by a short 200 ps equilibration. 


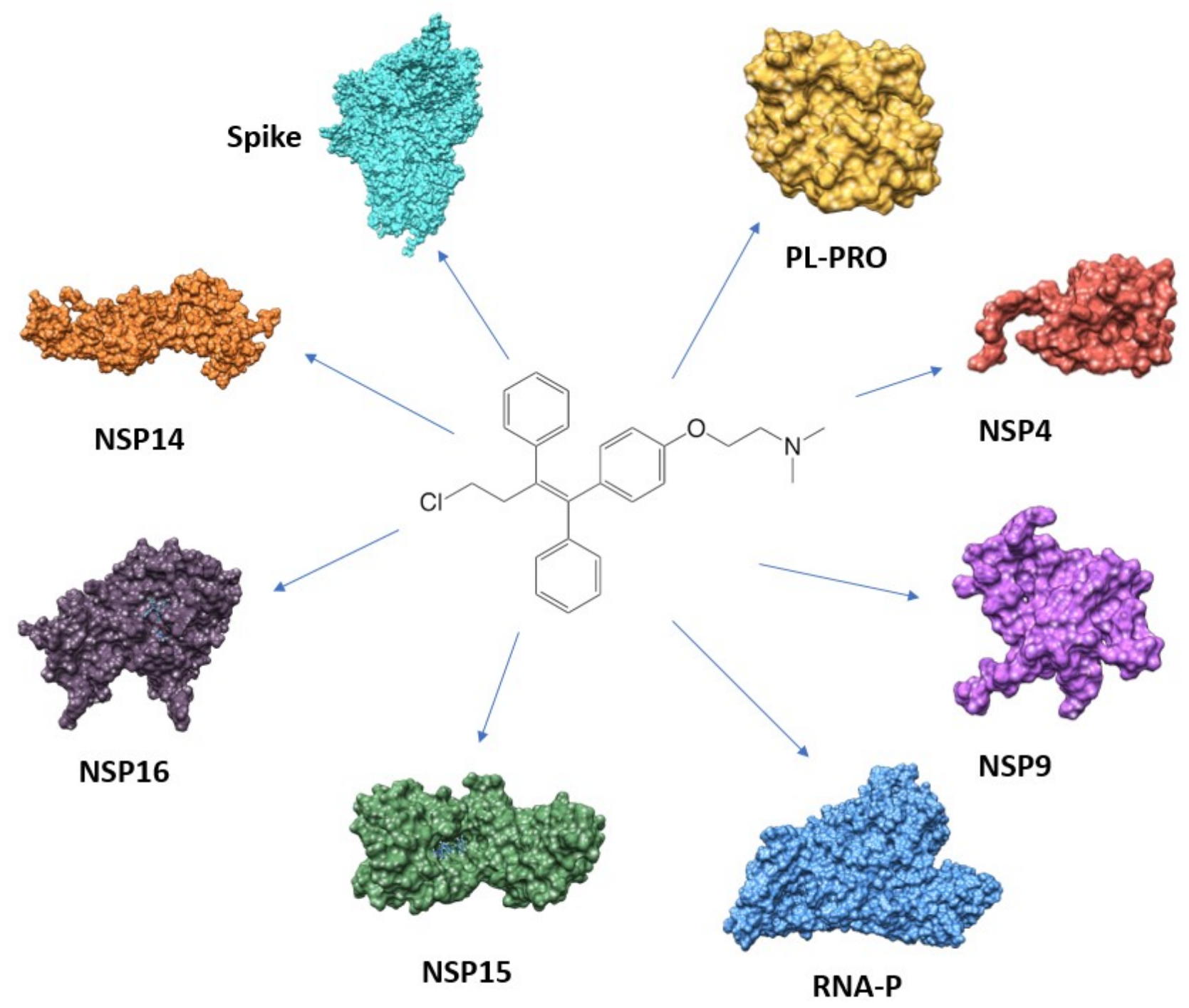

Figure 1: A diagram illustrating the workflow of computational identification of viral targets of toremifene across the SARS-CoV-2 proteome. Toremifene (center) with potential targets for molecular docking. From top right: PL-PRO, papain-like protease; NSP4, non-structural protein 4; NSP9, non-structural protein 9; RNA-P, RNAdirected RNA polymerase; NSP15, non-structural protein 15; NSP16, non-structural protein 16; NSP14, non-structural protein 14; Spike, spike glycoprotein. 


\subsection{Molecular Docking}

The toremifene structure was downloaded from the ZINC database ${ }^{13}$, while all crystal structures used for docking were obtained from the RCSB protein data bank ${ }^{20}$. All docking was done using AutoDock Vina ${ }^{21}$ within UCSF Chimera. The highest scoring binding pose $(\mathrm{kCal} / \mathrm{mol})$ was selected for further analysis where appropriate (Figure 1). Each search generated 10 binding modes with the exhaustiveness set to the maximum value. Proteins without a clear binding region (for example, accessory protein $7 a$ ) were not included. The search grid for each protein was selected to encompass the entire protein.

\subsection{Molecular Simulation System Construction}

Each system was constructed using the solution builder in CHARMM-GUI22-24. Following a processing step involving the addition of hydrogens not added in docking and parameterization of the ligand, a water box with edges at least $10 \AA$ from any part of the protein was added. The system was neutralized and brought to an ionic strength of 0.15 M using sodium and chloride ions. The CHARMM General Force Field (CGenFF) ${ }^{25}$ was used to parameterize toremifene, while the CHARMM36m force field was used to parameterize the protein, ions, and TIP3P water molecules.

\subsection{Simulation Parameters}

All systems were simulated using GROMACS 2020.1 on the AiMOS Supercomputer at the Rensselaer Polytechnic Institute Center for Computational Innovations in a three-step process. Initial minimization of the systems was run until changes in the potential energy 
of the system reached machine precision. Following minimization, an NVT equilibration step was completed with a 2 fs timestep for 500,000 steps using $400 \mathrm{~kJ} \mathrm{~mol}^{-1} \mathrm{~nm}^{-2}$ and $40 \mathrm{~kJ} \mathrm{~mol}^{-1} \mathrm{~nm}^{-2}$ positional restraints on the backbone and sidechains, respectively. A 500 ns production step was completed using the NPT ensemble with no position restraints and a 2 fs timestep.

Hydrogen atoms were constrained using the $\mathrm{LINCS}^{26}$ algorithm. Temperature for the system was held at $300 \mathrm{~K}$ using a Nose-Hoover thermostat ${ }^{27}$ with a 1 ps coupling constant. For the production simulation, pressure was coupled isotropically using a Parrinello-Rahman barostat ${ }^{28}$ with a 5.0 ps coupling constant and compressibility of $4.5 \mathrm{e}-$ 05 bar $^{-1}$ to maintain a pressure of 1 bar. The pair-list cutoff was constructed using the Verlet scheme ${ }^{29}$ with a cutoff distance of $1.2 \mathrm{~nm}$. Particle mesh Ewald electrostatics ${ }^{30}$ were used to describe coulombic interactions with a $1.2 \mathrm{~nm}$ cutoff, while van der Waals forces were smoothly switched to using between 1.0 and $1.2 \mathrm{~nm}$ using a force-switch modifier to the cut-off scheme. Linear center of mass translation was removed every 100 steps for the entire system.

\subsection{MM/PBSA calculations}

For systems which were chosen for further analysis, MM/PBSA (Molecular Mechanics / Poisson-Boltzmann Surface Area) calculations were done using g_mmpbsa ${ }^{31}$, a GROMACS tool used to calculate an estimated binding affinity. The final 50 nanoseconds of the trajectory are selected for the calculation, sampled every 200 picoseconds, for a 
total of 251 frames. MM/PBSA calculations were completed on the Pitzer supercomputer at the Ohio Supercomputer Center.

\section{RESULTS}

\subsection{Molecular Docking}

SARS-CoV-2 has a roughly $30 \mathrm{~kb}$ genome which encodes 29 proteins $^{32}$. These 29 proteins include 16 non-structural proteins (NSP), 4 structural proteins, and 9 accessory proteins. The non-structural proteins include proteinases (NSP3, NSP5), RNA polymerases (NSP12), helicases (NSP13), ribonucleases (NSP14, NSP15) and methyltransferases (NSP14, NSP16), while structural proteins are involved in viral assembly (envelope, membrane) and binding with the host protein (spike glycoprotein).

Molecular docking over the entire protein was carried out on 13 of the 29 possible viral proteins in SARS-CoV-2. These 13 were selected based on a combination of criteria: 1) Whether a crystal structure for the protein exists. 2) if a crystal structure does not exist, is there a template protein (generally from SARS-CoV-1) available to use for homology modeling. 3) Based on either the crystal structure or homology modeling, is there a potential binding pocket. Proteins which did not meet these criteria (for example, the membrane protein has partial homologous coverage at $\sim 20 \%$ homology; protein $3 a$ does not appear to have a potential binding pocket) were not chosen for the docking study. The best scoring poses are tabulated in Table 1, with a larger negative number indicating a better binding affinity. Unsurprisingly, the affinity was not high for a few of the smaller systems (NSP1 and the nucleocapsid, for example), which were included as a sort of 
negative control; it was not expected that good binding would be achieved with these systems. Interestingly, the spike glycoprotein and NSP14 had the highest binding affinities based on molecular docking.

Table 1: Docking results for toremifene with all potential targets.

\begin{tabular}{|c|c|c|c|}
\hline Protein & Model & Percent Homology & Affinity (kCal/mol) \\
\hline NSP1 & $2 \mathrm{HSX}^{33}$ & 86.1 & -5.1 \\
\hline PL-PRO & $6 \mathrm{VXS}$ & 100 & -6.6 \\
\hline NSP4 & $3 \mathrm{VCB}^{34}$ & 61.4 & -6 \\
\hline 3CL-PRO & $5 R 7 Y$ & 100 & -5.9 \\
\hline NSP7 & $6 \mathrm{M} 71^{35}$ & 100 & -5.7 \\
\hline NSP9 & $6 \mathrm{~W} 4 \mathrm{~B}$ & 100 & -5.7 \\
\hline RNA-P & $7 \mathrm{BTF}^{35}$ & 100 & -6.5 \\
\hline Helicase & $6 \mathrm{JYT}^{36}$ & 99.8 & -5.9 \\
\hline NSP14 & $5 \mathrm{C} 8 \mathrm{~S}^{37}$ & 95.1 & -7.2 \\
\hline NSP15 & $6 \mathrm{~W} 01^{38}$ & 100 & -6.1 \\
\hline NSP16 & $6 \mathrm{~W} 4 \mathrm{H}$ & 100 & -6.1 \\
\hline Spike & $6 V_{S B}^{39}$ & 100 & -6.9 \\
\hline Nucleocapsid & 6VYO & 100 & -5.6 \\
\hline
\end{tabular}

Note: All PDBs were downloaded from RSCB database: https://www.rcsb.org. Proteins with no potential binding region were not tested. Systems listed as $100 \%$ homology used the crystal structure as opposed to a homology model.

To determine which systems would be selected for further analysis via simulation to better determine if a particular protein-ligand binding pose maintains its integrity, we visually inspected the binding of each system. While a hard cutoff was not selected, certain systems were rejected for further analysis. Systems which did not have strong binding within a clear pocket were not simulated, including NSP1, NSP7, NSP9, the helicase, and the nucleocapsid. As an example, the interaction with NSP9 does not 
involve a binding pocket, but sits on the surface of the protein. We have no expectation that such an interaction would be maintained throughout a simulation, and therefore did not include it in the next step.

Table 2: Number of water molecules, ions, total number of atoms, and residence time of toremifene in the binding region as determined by molecular docking.

\begin{tabular}{lllll}
\hline Protein & TIP3P Water & $\mathbf{N a}^{+} / \mathbf{C l}^{-}$ & System Size & Residence Time \\
& & & & \\
\hline PL-PRO & 8912 & $26 / 26$ & 29387 & $34.4 \mathrm{~ns}$ \\
NSP4 & 12155 & $34 / 34$ & 38019 & $123.6 \mathrm{~ns}$ \\
3CL-PRO & 26898 & $79 / 76$ & 85553 & $284.2 \mathrm{~ns}$ \\
RNA-P & 75727 & $222 / 215$ & 242404 & $60.4 \mathrm{~ns}$ \\
NSP14 & 94844 & $270 / 272$ & 293433 & $500 \mathrm{~ns}$ \\
NSP15 & 25929 & $84 / 73$ & 83481 & $35.3 \mathrm{~ns}$ \\
NSP16 & 13133 & $37 / 41$ & 44220 & $11.5 \mathrm{~ns}$ \\
Spike & 277372 & $794 / 785$ & 885596 & $500 \mathrm{~ns}$ \\
\hline
\end{tabular}

Note: A time of 500 nanoseconds indicates the drug did not leave the binding pocket.

Please see the supplementary materials for details on proteins with residence time less than 500 nanoseconds.

\subsection{Molecular Dynamics}

Each of the above systems with a strong predicted binding was simulated according to the protocol listed in the methods. Here, we have monitored the protein-ligand interaction over the entire 500 nanosecond trajectory. Unsurprisingly, due to the low binding affinities, the interaction between the ligand and the protein was not maintained throughout the trajectory in most systems. Table 2 indicates the length of simulation before a particular protein-ligand system lost its interaction. 
Both the interaction between toremifene and NSP14, as well as that between toremifene and the spike glycoprotein, were maintained (and within the original binding pocket) throughout the entire 500 nanosecond trajectory. These two systems were analyzed further to determine the nature of the protein-ligand interactions.

To ensure the results were not simply a result of poor initial docking poses, all systems for which toremifene did not maintain contact with the initial docked region were redocked using SwissDock ${ }^{40}$ selecting the "accurate" setting under "extra parameters", with flexible residues within 5 angstroms of the docked ligand. A specific region of interest was not defined. These newly docked systems were also simulated in the same fashion as those docked with AutoDock, and yielded similar results; all simulations resulted in a residence time less than 200 nanoseconds.

\subsection{Toremifene interacts with the spike glycoprotein}

The initial docking position (Figure 2a) lies at the interface between two separate domains in the spike glycoprotein, with one domain having its receptor binding domain (RBD) in the "up" position, while the other having its RBD in the "down" position. In the B domain of PDB ID 6VSB, the interaction with the spike glycoprotein is with the helical region between the loop separating the $\mathrm{S} 1$ and $\mathrm{S} 2$ subunits and the fusion peptide, shown in SARS-CoV-1 to mediate membrane fusion in a calcium-dependent manner ${ }^{41}$, while the interaction in the A domain involves the $\mathrm{N}$-terminal region of the $\mathrm{RBD}$, as well as heptad repeat 1 (HR1), a key component of the fusion core (Figure $\mathbf{2 b}, \mathbf{4 a}$ ). While the non-helical linker between T941 and L945 did extend to K947 in the B and C domains, the remainder 
of this helical region remained unperturbed when compared to the crystal structure, with a long helix between L948 and S967. However, the interaction with toremifene resulted in a helical region between S943 and Q954, with a short helical region from A958 through T961 and the remainder unstructured. An interaction between the dimethylamine region of toremifene and Q954 and N955 appears to be key in perturbing the secondary structure of HR1.

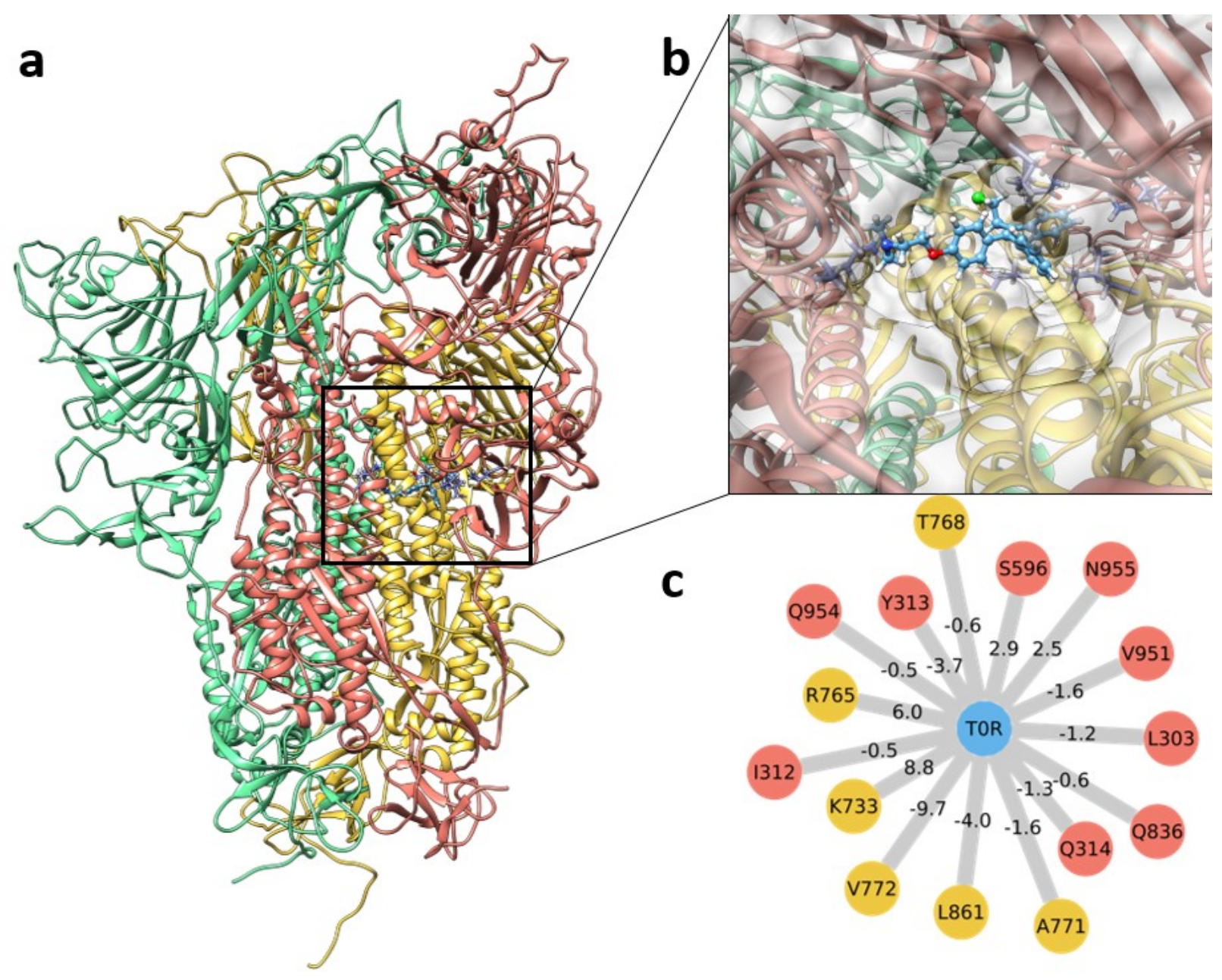

Figure 2: Toremifene likely interacts with the spike glycoprotein of SARS-CoV-2.

(a) The full-length crystal structure (PDB ID: 6VSB) of the homotrimeric spike glycoprotein with toremifene. (b) The final conformation of toremifene (blue, ball and 
stick) with the spike glycoprotein at 500 nanoseconds of simulation. (c) Key interacting residues with toremifene. Edges are labeled with MM/PBSA interaction energies.

In an effort to determine the strength of the interaction, we carried out an MM/PBSA binding affinity calculation between the entire protein and toremifene. Our calculation resulted in a final binding energy of $-91.036( \pm 0.933) \mathrm{kJ} / \mathrm{mol}$. The main contributors (Figure 2c) to this binding energy were V772 and L861 in the B domain due to large nonpolar interactions $(-9.7$ and $-4.0 \mathrm{~kJ} / \mathrm{mol}$, respectively), And Y313 in the A domain due to a chlorine-pi interaction $(-3.7 \mathrm{~kJ} / \mathrm{mol})$. The chlorine-pi interaction could indicate a potential mechanism by which toremifene has a stronger inhibitory action than tamoxifen as seen in SARS-CoV-1.

\subsection{Toremifene potentially displaces functional ligands in NSP14}

NSP14 has both exoribonuclease and methyl transferase activity; here, we have found a strong interaction with the N7-methyl transferase domain (Figure 3a). Throughout the molecular dynamics simulation, very little movement of the ligand was observed. The docked position appears as though it would potentially be inhibitory to interaction with the functional ligand S-adenosyl methionine, while clearly being inhibitory to interaction with its substrate, Gppp-RNA (Figure 3c,4b). As with the spike protein, we assessed the binding affinity using MM/PBSA, finding a significant pi-pi interaction with F426 (-8.0 $\mathrm{kJ} / \mathrm{mol})$, a chlorine-pi interaction with F506 $(5.0 \mathrm{~kJ} / \mathrm{mol})$, a strong hydrophobic interaction with C309 $(-4.2 \mathrm{~kJ} / \mathrm{mol})$, and a total binding energy of $-119.805( \pm 1.013) \mathrm{kJ} / \mathrm{mol}$. Many 
of the residues identified here as interacting with toremifene (Figure 3b) were identified in the crystal structure used to generate our homology model as interacting with GpppA.

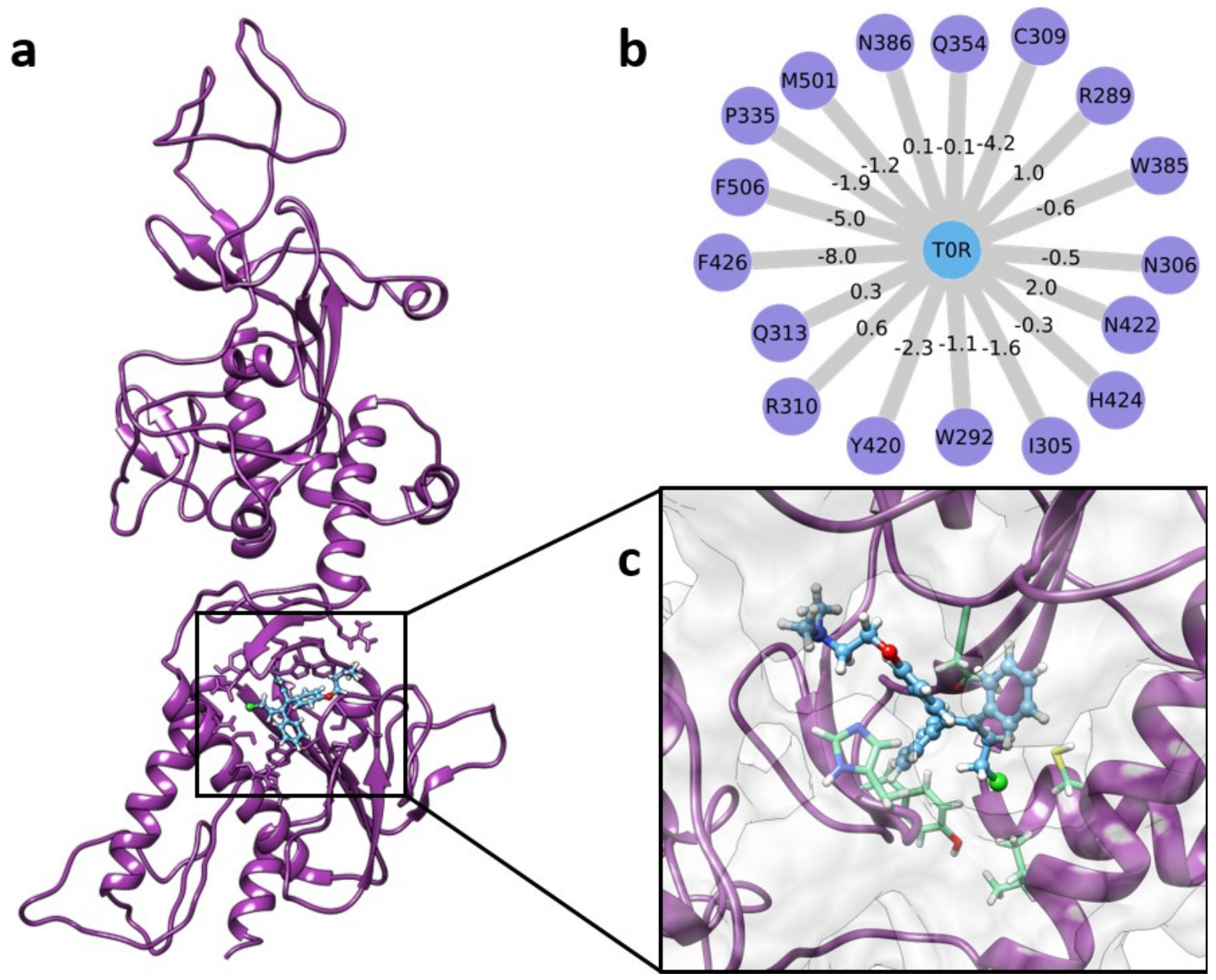

Figure 3: Toremifene likely displaces functional ligands in NSP14 in SARS-CoV-2.

(a) The homology model of NSP14 with toremifene. (b) Key interacting residues with toremifene, with edges labeled with MM/PBSA interaction energy. (c) Final pose of toremifene (blue, ball and stick) with NSP14 at 500 nanoseconds of simulation. The RMSD for all simulations can be found in Supplementary Figure 7. 


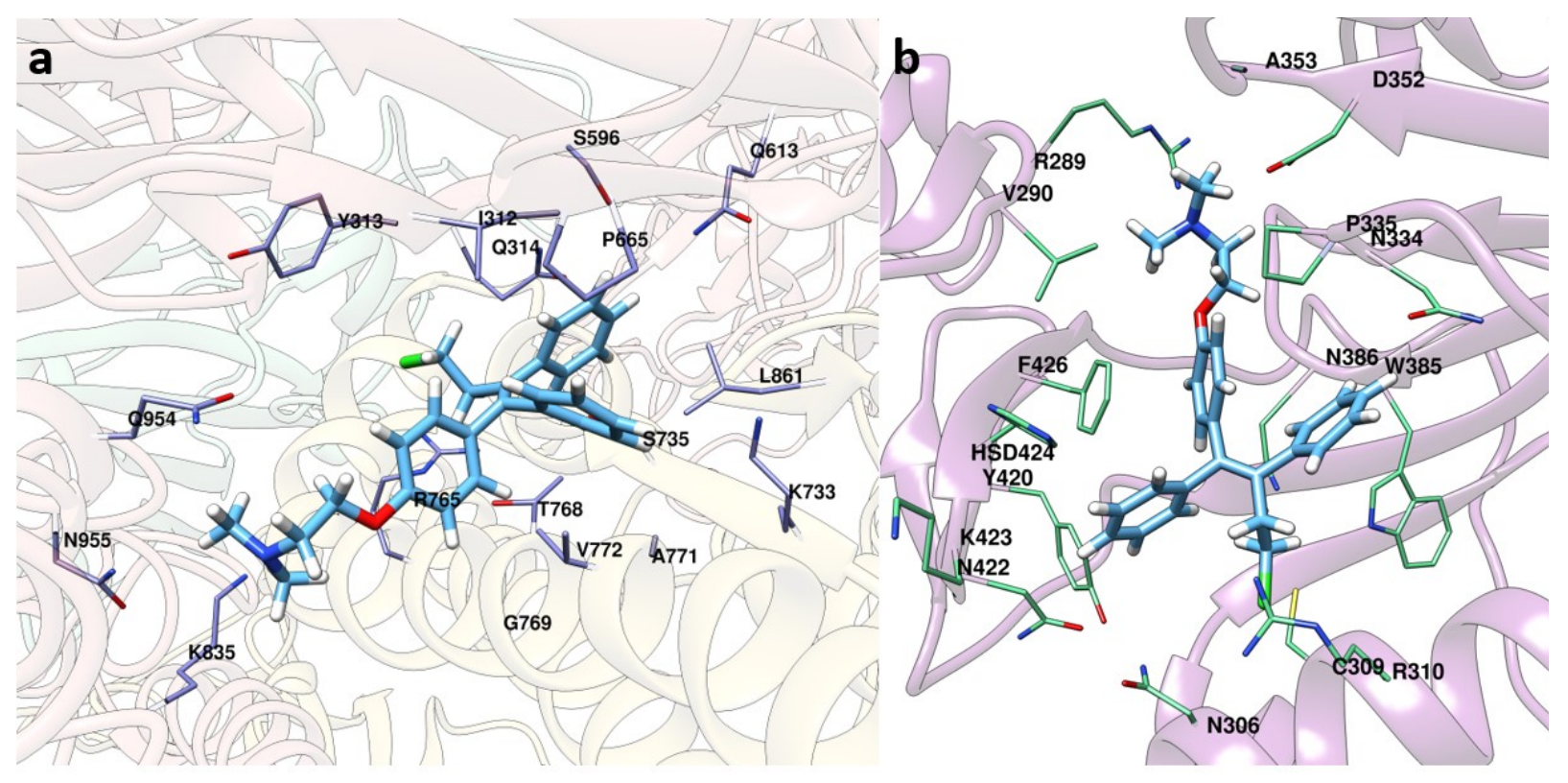

Figure 4: Toremifene interacts with the spike glycoprotein and NSP14. Stick representations of the final pose at 500 nanoseconds for toremifene with (a) the spike glycoprotein and (b) NSP14. Wireframe residues represented in each image are within 3.0 angstroms of toremifene in the final frame of the 500 nanosecond simulation.

\section{DISCUSSION}

We have demonstrated two plausible targets for toremifene in SARS-CoV-2. Previous work has indicated that, as noted earlier, toremifene is likely to be inhibitory to viral entry. A proposed mechanism for ebola posited a mechanism by which fusion between the viral and endosomal membranes is disrupted. The interaction with the spike glycoprotein proposed here could prevent such a fusion through its disruption of the HP1 helix. The interaction with NSP14 elucidated here would indicate an inhibition of viral reproduction by interfering with interaction with the substrate. 
Toremifene, a first generation nonsteroidal SERM, shows striking activity in blocking multiple viral infections, including Ebola ${ }^{5,6}\left(\mathrm{IC}_{50}=\sim 1 \mu \mathrm{M}\right), \mathrm{MERS}-\mathrm{CoV}^{4}\left(\mathrm{EC}_{50}=\right.$ $12.9 \mu \mathrm{M})$, SARS-CoV- $1^{4}\left(\mathrm{EC}_{50}=11.97 \mu \mathrm{M}\right)$, and SARS-CoV-2 ${ }^{8}\left(\mathrm{IC}_{50}=3.58 \mu \mathrm{M}\right)$, in established, virus infected human cell lines. The inhibition in Ebola by SERMs has been found to not be via the estrogen receptor pathway, but instead disrupt endolysosomal calcium, leading to accumulation and thereby blocking Ebola entry ${ }^{42}$. Toremifene has been approved for the treatment of breast cancer for over 25 years ${ }^{43}$, and has been investigated as a treatment for prostate cancer ${ }^{44}$. In this study, we have determined two potential targets for toremifene, the spike glycoprotein and NSP14. The combination of consistent findings from cell line data supporting the antiviral effects of toremifene against coronaviruses, along with reasonable tolerability, provide the basis to pursue toremifene as a viable candidate therapeutic against SARS-CoV-2.

Caution, however, must be exercised when interpreting these results. It should be noted that, to this date, there are no crystal structures published for the spike glycoprotein with an inhibitory ligand. Similarly, no inhibitors have been crystallized with NSP14, nor has a structure for NSP14 in SARS-CoV-2 been crystallized, though the homology with SARS-CoV-1 is indeed high. However, work on the flavivirus methyltransferase ${ }^{38}$ has indicated there is a potential for inhibition. While the proposed mechanisms for SARSCoV-1 in toremifene are posited to not involve human proteins, further study would be required to confirm the mechanism. While in vitro studies on toremifene do exist for SARS-CoV-2, there are no studies to date which allow a comparison with other SERMs, as has been done with SARS-CoV-1 and MERS-CoV. 
Future work will be needed to confirm these results; optimally, the determination of a co-crystal structure with NSP14 and/or the spike glycoprotein from SARS-CoV-2 with toremifene would be solved. Alternatively, other functional validation analyses could be carried out to determine the correct protein target. The final poses could also be used to inform further work to refine a potential inhibitor using medicinal chemistry techniques.

\section{CONCLUSION}

The present study has demonstrated two potential targets for toremifene in SARS-CoV2. We have demonstrated a potential mechanism for inhibition via the spike glycoprotein, through which an interaction with Q954 and N955 in heptad repeat 1 appears to disturb the secondary structure, resulting in what is normally a long alpha helix instead having a lack of secondary structure. Additionally, the interaction we've found with NSP14 appears as though it could be inhibitory in two ways: First, it appears as though there would be steric hindrance between toremifene and any functional ligands (such as S-adenosyl methionine), as well as interfering with substrate interaction in the catalytic pocket. Further experimental and functional studies would be needed to validate these findings, such as a co-crystal structure of the spike glycoprotein or NSP14 with toremifene.

\section{Acknowledgements}

We acknowledge support from the Oak Ridge Leadership Computing Facility.

Author contributions: F.C. conceived the study. W.R.M. performed all experiments and data analysis. W.R.M. and F.C. wrote and critically revised the manuscript. 
Competing interests: The authors declare that they have no conflict of interest. The content of this publication does not necessarily reflect the views of the Cleveland Clinic.

\section{REFERENCES}

1. Cheng, F., Murray, J. L. \& Rubin, D. H. Drug Repurposing: New Treatments for Zika Virus Infection? Trends in Molecular Medicine vol. 22 919-921 (2016).

2. Cheng, F. et al. Systems Biology-Based Investigation of Cellular Antiviral Drug Targets Identified by Gene-Trap Insertional Mutagenesis. PLOS Computational Biology 12, e1005074 (2016).

3. Zhou, Y. et al. Network-based drug repurposing for novel coronavirus 2019nCoV/SARS-CoV-2. Cell Discovery 6, 14 (2020).

4. Dyall, J. et al. Repurposing of clinically developed drugs for treatment of Middle East respiratory syndrome coronavirus infection. Antimicrobial Agents and Chemotherapy 58, 4885-4893 (2014).

5. Johansen, L. M. et al. FDA-approved selective estrogen receptor modulators inhibit ebola virus infection. Science Translational Medicine 5, 190 ra79 (2013).

6. Zhao, Y. et al. Toremifene interacts with and destabilizes the Ebola virus glycoprotein. Nature 535, 169-172 (2016).

7. $\mathrm{Si}$, L. et al. Human organs-on-chips as tools for repurposing approved drugs as potential influenza and COVID19 therapeutics in viral pandemics. bioRxiv 2020.04.13.039917 (2020) doi:10.1101/2020.04.13.039917. 
8. Jeon, S. et al. Identification of antiviral drug candidates against SARS-CoV-2 from FDA-approved drugs. Antimicrobial Agents and Chemotherapy (2020) doi:10.1128/AAC.00819-20.

9. ul Qamar, M. T., Alqahtani, S. M., Alamri, M. A. \& Chen, L.-L. Structural basis of SARS-CoV-2 3CLpro and anti-COVID-19 drug discovery from medicinal plants. Journal of Pharmaceutical Analysis (2020) doi:10.1016/j.jpha.2020.03.009.

10. Chen, Y. W., Yiu, C.-P. B. \& Wong, K.-Y. Prediction of the SARS-CoV-2 (2019nCoV) 3C-like protease (3CLpro) structure: virtual screening reveals velpatasvir, ledipasvir, and other drug repurposing candidates. F1000Research 9, 129 (2020).

11. Dai, W. et al. Structure-based design of antiviral drug candidates targeting the SARS-CoV-2 main protease. Science eabb4489 (2020) doi:10.1126/science.abb4489.

12. Arya, R., Das, A., Prashar, V. \& Kumar, M. Potential inhibitors against papainlike protease of novel coronavirus (SARS-CoV-2) from FDA approved drugs. doi:10.26434/chemrxiv.11860011.v2.

13. Irwin, J. J., Sterling, T., Mysinger, M. M., Bolstad, E. S. \& Coleman, R. G. ZINC: A Free Tool to Discover Chemistry for Biology. Journal of Chemical Information and Modeling 52, 1757-1768 (2012).

14. Parks, J. M. \& Smith, J. C. How to Discover Antiviral Drugs Quickly. New England Journal of Medicine NEJMcibr2007042 (2020) doi:10.1056/NEJMcibr2007042. 
15. Altschul, S. F. et al. Gapped BLAST and PSI-BLAST: a new generation of protein database search programs. Nucleic acids research 25, 3389-3402 (1997).

16. Pettersen, E. F. et al. UCSF Chimera-A visualization system for exploratory research and analysis. Journal of Computational Chemistry 25, 1605-1612 (2004).

17. Eswar, N. et al. Comparative Protein Structure Modeling Using Modeller. Current protocols in bioinformatics / editoral board, Andreas D.Baxevanis ... [et al.] 0 5, Unit$5.6(2006)$.

18. Abraham, M. J. et al. GROMACS: High performance molecular simulations through multi-level parallelism from laptops to supercomputers. SoftwareX1-2, 1925 (2015).

19. Best, R. B. et al. Optimization of the Additive CHARMM All-Atom Protein Force Field Targeting Improved Sampling of the Backbone $\phi, \psi$ and Side-Chain X 1 and X 2 Dihedral Angles. Journal of Chemical Theory and Computation 8, 3257-3273 (2012).

20. Berman, H. M. et al. The Protein Data Bank. Nucleic acids research 28, 235-242 (2000).

21. Trott, O. \& Olson, A. J. AutoDock Vina: improving the speed and accuracy of docking with a new scoring function, efficient optimization, and multithreading. Journal of computational chemistry 31, 455-61 (2010). 
22. Kim, S. et al. CHARMM-GUI ligand reader and modeler for CHARMM force field generation of small molecules. Journal of Computational Chemistry 38, 1879-1886 (2017).

23. Lee, J. et al. CHARMM-GUI Input Generator for NAMD, GROMACS, AMBER, OpenMM, and CHARMM/OpenMM Simulations Using the CHARMM36 Additive Force Field. Journal of Chemical Theory and Computation 12, 405-413 (2016).

24. Jo, S., Kim, T., Iyer, V. G. \& Im, W. CHARMM-GUI: A web-based graphical user interface for CHARMM. Journal of Computational Chemistry 29, 1859-1865 (2008).

25. Vanommeslaeghe, K. et al. CHARMM general force field: A force field for druglike molecules compatible with the CHARMM all-atom additive biological force fields. Journal of Computational Chemistry 31, 671-690 (2010).

26. Hess, B., Bekker, H., Berendsen, H. J. C. \& Fraaije, J. G. E. M. LINCS: A linear constraint solver for molecular simulations. Journal of Computational Chemistry $\mathbf{1 8 ,}$ 1463-1472 (1997).

27. Braga, C. \& Travis, K. P. A configurational temperature Nosé-Hoover thermostat. The Journal of Chemical Physics 123, 134101 (2005).

28. Parrinello, M. \& Rahman, A. Polymorphic transitions in single crystals: A new molecular dynamics method. Journal of Applied Physics 52, 7182-7190 (1981).

29. Verlet, L. Computer \&quot;Experiments\&quot; on Classical Fluids. I. Thermodynamical Properties of Lennard-Jones Molecules. Physical Review 159, 98-103 (1967). 
30. Darden, T., York, D. \& Pedersen, L. Particle mesh Ewald: An $N \cdot \log (N)$ method for Ewald sums in large systems. The Journal of Chemical Physics 98, 1008910092 (1993).

31. Kumari, R., Kumar, R., Lynn, A. \& Lynn, A. g_mmpbsa —A GROMACS Tool for High-Throughput MM-PBSA Calculations. Journal of Chemical Information and Modeling 54, 1951-1962 (2014).

32. Kim, D. et al. The Architecture of SARS-CoV-2 Transcriptome. (2020) doi:10.1016/j.cell.2020.04.011.

33. Almeida, M. S., Johnson, M. A., Herrmann, T., Geralt, M. \& Wüthrich, K. Novel $\beta$-Barrel Fold in the Nuclear Magnetic Resonance Structure of the Replicase Nonstructural Protein 1 from the Severe Acute Respiratory Syndrome Coronavirus. Journal of Virology 81, 3151-3161 (2007).

34. Xu, X. et al. Crystal Structure of the C-Terminal Cytoplasmic Domain of NonStructural Protein 4 from Mouse Hepatitis Virus A59. PLoS ONE 4, e6217 (2009).

35. Gao, Y. et al. Structure of the RNA-dependent RNA polymerase from COVID-19 virus. Science eabb7498 (2020) doi:10.1126/science.abb7498.

36. Jia, Z. et al. Delicate structural coordination of the Severe Acute Respiratory Syndrome coronavirus Nsp13 upon ATP hydrolysis. Nucleic acids research 47, $6538-6550$ (2019). 
37. Ma, Y. et al. Structural basis and functional analysis of the SARS coronavirus nsp14-nsp10 complex. Proceedings of the National Academy of Sciences of the United States of America 112, 9436-9441 (2015).

38. Kim, Y. et al. Crystal structure of Nsp15 endoribonuclease NendoU from SARSCoV-2. Protein Science pro.3873 (2020) doi:10.1002/pro.3873.

39. Wrapp, D. et al. Cryo-EM structure of the 2019-nCoV spike in the prefusion conformation. Science (New York, N.Y.) 367, 1260-1263 (2020).

40. Grosdidier, A., Zoete, V. \& Michielin, O. SwissDock, a protein-small molecule docking web service based on EADock DSS. Nucleic Acids Research 39, W270W277 (2011).

41. Lai, A. L., Millet, J. K., Daniel, S., Freed, J. H. \& Whittaker, G. R. The SARS-CoV Fusion Peptide Forms an Extended Bipartite Fusion Platform that Perturbs Membrane Order in a Calcium-Dependent Manner. Journal of Molecular Biology 429, 3875-3892 (2017).

42. Fan, H. et al. Selective inhibition of Ebola entry with selective estrogen receptor modulators by disrupting the endolysosomal calcium OPEN. Nature Publishing Group (2017) doi:10.1038/srep41226.

43. Mustonen, M. V., Pyrhönen, S. \& Kellokumpu-Lehtinen, P. L. Toremifene in the treatment of breast cancer. World Journal of Clinical Oncology vol. 5 393-405 (2014). 
44. Fujimura, T. et al. Toremifene, a selective estrogen receptor modulator, significantly improved biochemical recurrence in bone metastatic prostate cancer: a randomized controlled phase II a trial. BMC Cancer 15, 836 (2015). 